\title{
Mars Atmospheric Entry Integrated Navigation with Partial Intermittent Measurements
}

\author{
Tai-shan Lou, ${ }^{1}$ Yan Wang, ${ }^{1}$ Guo-qiang Ding, ${ }^{1}$ and Liang-yu Zhao ${ }^{2}$ \\ ${ }^{1}$ School of Electrical and Information Engineering, Zhengzhou University of Light Industry, Zhengzhou 45002, China \\ ${ }^{2}$ School of Aerospace Engineering, Beijing Institute of Technology, Beijing 100081, China \\ Correspondence should be addressed to Tai-shan Lou; tayzan@sina.com
}

Received 12 June 2017; Accepted 13 August 2017; Published 24 September 2017

Academic Editor: Christopher J. Damaren

Copyright (C) 2017 Tai-shan Lou et al. This is an open access article distributed under the Creative Commons Attribution License, which permits unrestricted use, distribution, and reproduction in any medium, provided the original work is properly cited.

\begin{abstract}
Signal degradation suffered by the vehicle is a combination brownout and blackout during Mars atmospheric entry. The communications brownout means that signal fades and blackout means that the signal is lost completely. The communications brownout and blackout periods are analyzed and predicted with an altitude and velocity profiles. In the brownout period, the range measurements between the vehicle and the orbiters are modeled as intermittent measurements with the radio signal arrival probabilities, which are distributed as a Rayleigh distribution of the electron number density around the entry vehicle. A new integrated navigation strategy during the Mars atmospheric entry phase is proposed to consider the probabilities of the radio measurements in the communications brownout and blackout periods under the IMU/beacon scenario based on the information filter with intermittent measurements. Numerical navigation simulations are designed to show the performance of the proposed navigation strategy under the integrated navigation scenario.
\end{abstract}

\section{Introduction}

On 5 August 2012, the Mars Science Laboratory's (MSL) Curiosity rover successfully landed on Mars after suffering the Seven Minutes of Terror, in which the MSL entry, descent, and landing (EDL) system brings the velocity of the vehicle from about $5.9 \mathrm{~km} / \mathrm{s}$ to $0.75 \mathrm{~m} / \mathrm{s}$. Especially, during Mars entry phase, the vehicle experienced the most rugged aerodynamic environment, which includes high temperature and pressure, peak heating (peak temperatures of up to $2090^{\circ} \mathrm{C}$ ), and peak deceleration (out at $15 \mathrm{~g}$ ), and the signal between the vehicle and the orbiters can fade or suffer from total loss to make the ultrahigh frequency (UHF) relay links from the MSL to the orbiters suffer a period of about $70 \mathrm{~s}$ of degradation $[1,2]$. The Mercury, Gemini, and Apollo spacecraft entering the Earth's atmosphere and the Mars Pathfinder entering Mars' atmosphere all endured communications blackouts, and the lasting time is from 30 seconds to several minutes [3]. During communications blackout period, it is difficult to perform real-time communication between the vehicle and the orbiter to provide the range measurements in time for the navigation system. So, it is necessary to predict trends or likelihood of the signal degradation and design appropriate navigation technology and strategy.

The communications blackout is caused by reflecting or absorbing the electromagnetic at some frequencies by the sheath of ionized atmospheric gases around the spacecraft when the spacecraft enters into a planetary atmosphere with a hypersonic velocity. Whether or not the communications blackout occurs depends on the electron number density (END), the critical electron number density (CEND) at the link frequency, such as UHF. If the electron number density exceeds the corresponding critical electron number density, the communications blackout may happen under some frequencies. In order to analyze and forecast any possible blackout problem, two aerothermodynamic analysis programs, which include the Jet Propulsion Laboratory (JPL) Normal Shock and Chemical Equilibrium Program (also called the Horton program) [4] and the Langley Aerothermodynamic Upwind Relaxation Algorithm (LAURA) program [5], have been used in Mars entry vehicles as they fly through Mars 
atmospheric environment [6]. The JPL Horton program was developed in the 1960s [4] and then used to predict any possible communications blackout problem $[2,6]$. These tools output the velocity of the shock layer and the electron number density at the stagnation point by using the composition, pressure, temperature, and density of the Mars atmosphere [6]. Morabito analyses the communications blackout problem of the Mars Pathfinder at X-band based on the wake-region electron density estimates produced by the JPL Horton program and the LAURA program and compares the predicted results of the two programs [6]. Morabito and Edquist predicted the communications blackout at UHF for different MSL entry cases by using the above two tools [3]. After the MSL successfully landed on Mars, Morabito and Edquist analyzed the UHF communications brownout and blackout experienced by the MSL during Mars atmospheric entry, which coincided with the predicted signal degradation from preflight analyses in literature [3]. Schratz et al. discussed the performance of the MSL telecommunications for UHF during the EDL phase and analyzed the signal strength and the plasma blackout during Mars entry [7].

Future Mars explorations need the capability of pinpoint landing on the preselected high scientifically valuable regions to meet the scientists' requirements. It is difficult to achieve this target if the only available measurement sensor is the on-board default inertial measurement unit (IMU) to provide measurement information for the navigation, guidance, and control system during Mars atmospheric entry phase $[8,9]$. An innovative integrated navigation scheme during Mars atmospheric entry was proposed, which is adding the range measurements between the vehicle and the surface beacons or orbiters by using the UHF radio communication $[10,11]$. The key technology of the above navigation scheme is that the UHF radio communication is not blocked by the plasma sheath around the vehicle. National Aeronautics and Space Administration (NASA) develops an advanced Mars Network's Electra UHF transceiver, which is a prototype, embedded, real-time navigation system and can provide radio communication information between the vehicle and an orbiter or a surface beacon [10, 12]. Levesque and de Lafontaine researched four integrated navigation scenarios and their observability based on radio ranging by using the unscented Kalman filter during the Mars atmospheric entry [11]. Li et al. did a lot of works based on a six-dimension dynamic model by using radio communication under the IMU/beacon integrated navigation schemes $[9,13,14]$. Yu et al. primarily analyzed the optimization of the Mars entry navigation beacon configuration based on the system observability by using radiometric measurements $[15,16]$. Lou et al. considered the uncertain model parameters and the measurement biases by using consider Kalman filter (also called Schmidt-Kalman filter) [17-19] and analyzed the sensitivity of the parameter uncertainties based on the robust desensitized extended Kalman filter [20] under the radio beacon navigation schemes during Mars atmospheric entry.

Morabito et al. [2] pointed that the UHF radio signals fade or break off in the communications brownout and blackout periods for the MSL during Mars atmospheric entry. This signal degradation makes the two-way range measurements for the navigation system cannot reach (or at a certain probability) the MSL. Li and Peng primarily analyzed the effect of the communications blackout under the radio beacons/IMU integrated navigation and pointed that as the communications blackout occurs, the navigation errors will gradually increase [9]. Wang and Xia compared the performance of the unscented Kalman filter under the surface beacons/IMU integrated navigation and the orbiters/IMU integrated navigation when the communications blackout happens [21]. The above researches considered that the vehicle does not receive any radio signal in the communications brownout period during Mars atmospheric entry and the probabilities of the arrival of the range measurements are not analyzed [2]. Adding the potential range measurements, which arrives at a certain probability, into the navigation system undoubtedly improves the navigation accuracy in the communications brownout period.

Recently, the intermittent measurements, which are also called missing measurements and modeled by a Bernoulli distributed sequence [22-24], were introduced into a series of filtering algorithms to deal with these intermittent data phenomena, such as the Kalman filtering [24, 25], unscented Kalman filter $[25,26]$, and robust filtering [23, 27]. These filters are committed to obtain the optimal estimates when the arrival of the measurement is not in time; in other words, the measurement is lost at a certain probability. This filtering problem with intermittent measurements has motivated researcher's interest, because these missing measurement phenomena are more popular in practical applications, such as the navigation of a moving vehicle [28], and networked control systems [29]. Subsequently, the intermittent measurement problem with different loss probabilities, which means that the measurement loss might be partial, has been investigated, and some solutions are proposed [30, 31].

The aim of this paper is to develop an integrated navigation scenario with the probabilities of the radio measurements in the communications brownout and blackout periods during the Mars atmospheric entry phase by using the information filter with intermittent measurements (IFIM). The communications brownout and blackout periods are analyzed and predicted with an altitude and velocity profiles during Mars atmospheric entry. The signal arrival probabilities of the UHF radio signals are modeled as the Rayleigh distribution of the electron number density around the entry vehicle. The range measurements between the vehicle and the orbiters are described as a Bernoulli distributed sequence with different probabilities in the communications brownout period. Then, the IFIM algorithm is derived and designed for the navigation filter. The numerical navigation simulations are designed to show the performance of the proposed navigation strategy.

This paper is organized as follows: firstly, the Mars entry dynamic model and measurement model based on the IMU and three orbiters are introduced; secondly, the communications brownout and blackout phenomena are predicted by an aerodynamical tool during Mars atmospheric entry; thirdly, the information filter with intermittent measurements is derived; and lastly, the results of the navigation simulation are discussed. 


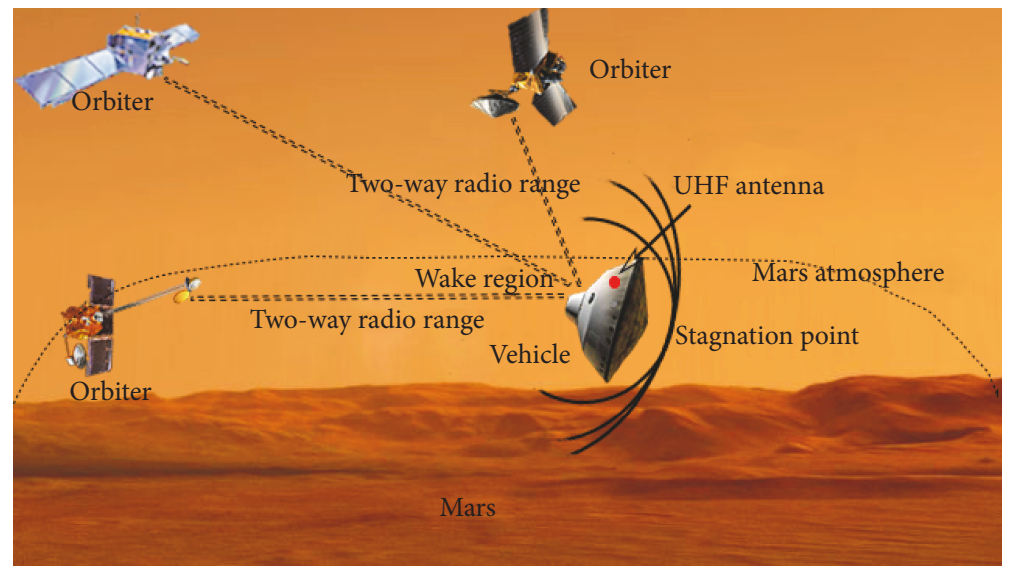

FIGURE 1: Diagram of three orbiter navigation scenarios, stagnation point, and wake region.

\section{Dynamics of the Navigation System}

2.1. Dynamic Equations for Mars Entry. During Mars atmospheric entry, the dynamic model of the vehicle is modeled as six state equations of a six-dimensional state, which includes the altitude $r$ (radial distance from the center of the vehicle mass to the Mars center), radial velocity $v$, flight path angle (FPA) $\gamma$, longitude $\theta$, latitude $\lambda$, and azimuth angle $\psi$ (a clockwise rotation angle starting at due north), in the Mars-centered Mars-fixed coordinate system. Some assumptions are that the planet is nonrotating, its atmosphere is stationary and quiet, and winds in the atmosphere and the centripetal and Coriolis effects are neglected $[17,32]$. Then, the six entry dynamic equations of the vehicle are described by $[11,17,19,32]$

$$
\begin{aligned}
\dot{r} & =v \sin \gamma, \\
\dot{v} & =-D-g \sin \gamma, \\
\dot{\gamma} & =\frac{1}{v}\left[\left(\frac{v^{2}}{r}-g\right) \cos \gamma+L \cos \phi\right], \\
\dot{\theta} & =\frac{v \cos \gamma \sin \psi}{r \cos \lambda}, \\
\dot{\lambda} & =\frac{v \cos \gamma \cos \psi}{r}, \\
\psi & =\frac{1}{v \cos \gamma}\left[\frac{v^{2}}{r} \sin \psi \cos ^{2} \gamma \tan \lambda+L \sin \phi\right],
\end{aligned}
$$

where $\phi$ is the bank angle, $g=\mu / r^{2}$ is the simplified gravitational acceleration, and $\mu$ is the Mars gravitational constant $\left(\mu=42,828.29 \times 10^{9} \mathrm{~m}^{3} / \mathrm{s}^{2}\right)$. $D$ and $L$ are, respectively, the aerodynamic drag and lift accelerations given as follows:

$$
\begin{aligned}
& D=\frac{1}{2} \rho v^{2} \frac{C_{D} S}{m}, \\
& L=\frac{1}{2} \rho v^{2} \frac{C_{L} S}{m},
\end{aligned}
$$

where $C_{D}$ and $C_{L}$ are, respectively, the vehicle drag coefficient and lift coefficient, $S$ is the reference surface area of vehicle, $m$ is the mass of the vehicle. $\rho$ represents the Mars atmospheric density with an exponential model is given by $[18,33]$

$$
\rho=\rho_{0} \exp \left\{\frac{r_{0}-r}{h_{s}}\right\}
$$

where $\rho_{0}$ is the nominal reference density on the Mars surface, $r_{0}$ denotes the nominal reference radial position $\left(r_{0}=3,437.2 \mathrm{~km}\right)$, and $h_{s}$ denotes the constant scale height $\left(h_{s}=7.5 \mathrm{~km}\right)$. For convenience's sake in this work, the liftto-drag ratio is defined by $L / D=C_{L} / C_{D}$, and the ballistic coefficient is defined as $B=C_{D} S / m$.

2.2. Measurement Models. To improve the navigation accuracy based on the IMU information, a potential integrated navigation scenario with both IMU and UHF radio communication is proposed to support pinpoint landing accuracy for future Mars missions. The IMU provides three components of acceleration for the navigation system. The UHF radio provides communication between the entry vehicle and the orbiting satellites or surface beacons (e.g., a preset fixed beacon or a previous Mars lander) and measures the distance between the vehicle and an orbiter or a surface beacon for the navigation system $[12,18,19,34]$. At present, there are only three available Mars orbiters to provide radio communications, which are Mars Reconnaissance Orbiter (MRO), Mars Odyssey (MO), and Mars Express (MEX), and no surface radio beacons are preset on Mars. In this work, three orbiting radio satellites are considered to provide the two-way range measurements by using the UHF radio communications. The diagram of the three orbiter navigation scenarios and the position of the UHF antenna, the stagnation point and the wake-region, which need to estimate the electron number density, is shown in Figure 1.

2.2.1. IMU Measurements. The IMU is used to measure the specific force components along three orthogonal axes and the angular rates about the axes by using the accelerometers 
and gyroscopes. Three components of acceleration $\tilde{a}$ are given by

$$
\tilde{a}=a+b_{a}+\eta_{a}
$$

where $a$ is the true linear acceleration along body axes, $b_{a}$ is the drift-rate biases of the accelerometer, and $\eta_{a}$ is assumed as zero-mean white Gaussian noise. In this work, the accelerometer measurement model is defined by

$$
a=\left[\begin{array}{lll}
-D & -L \sin \phi & L \cos \phi
\end{array}\right]^{T} .
$$

2.2.2. Range Measurements. The UHF radio communications provide the two-way range measurements between the vehicle and the orbiters within sight. The two-way range measurement $\tilde{R}$ in the Mars-centered inertial coordinate system is reconstructed by

$$
\tilde{R}=\sqrt{\left(\mathbf{r}-\mathbf{r}_{o}\right)^{\mathrm{T}}\left(\mathbf{r}-\mathbf{r}_{o}\right)}+\xi_{R}
$$

where $\mathbf{r}$ is the position vector of the entry vehicle, which is calculated by

$$
\mathbf{r}_{l}=\mathbf{r}[\cos \lambda \cos \theta, \cos \lambda \sin \theta, \sin \lambda]^{T},
$$

$\mathbf{r}_{o}$ is the position vector of the orbiter, and $\xi_{R}$ is the range measurement noise. In this work, three orbiters within sight are utilized to provide the range measurements for the navigation system, and the corresponding parameters of the three orbiters are listed in the following section.

\section{Communications Blackout Estimations and Signal Loss Probabilities during Mars Entry}

The UHF radio communications between the vehicle and an orbiter or a surface beacon during Mars entry can become disrupted, when the electron number density of the ionized particles around the hypersonic vehicle increases sufficiently highly. The intervening charged particles can reflect or absorb the radio signal energy to degrade the quality of the communications, such as brownout or blackout. The communications brownout/blackout problem can decrease the sources of the navigation measurement and make the measurement model with range information mismatch $[6,22]$. The navigation accuracy during Mars entry will greatly degrade or even make unexpected results. So, it is necessary to analyze the degree of the signal degradation and the effect of the different signal attenuations for the communication.

3.1. Effects of Charged Particles on Communications with $U H F$. The communication signal between the entry vehicle and the preselected radio beacons will be attenuated, because the surrounding plasma on the vehicle during Mars atmospheric entry will absorb and reflect the signal if the plasma density is sufficiently great. Spencer points that the main matters to reflect waves in a plasma gas are electrons, and the principle factors to determine the signal propagation condition are the transmission frequency and the plasma frequency of the charged particle sheath $[35,36]$.

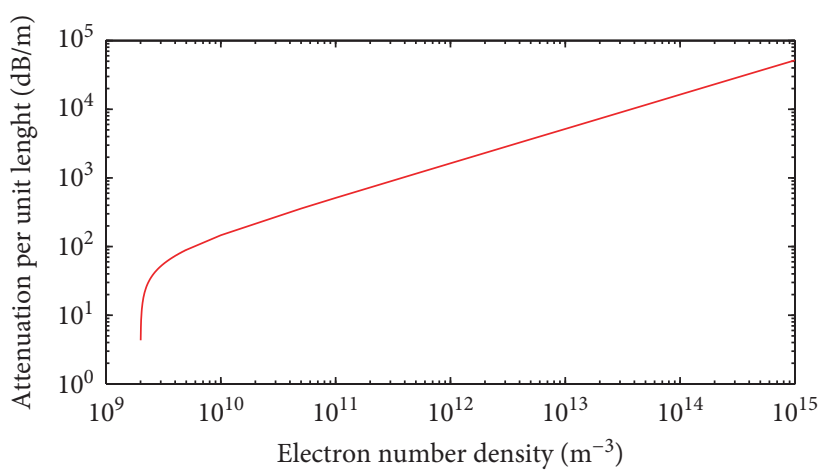

FIgURE 2: Attenuation per unit length versus electron number density for UHF.

During Mars atmospheric entry, the heating of atmospheric gases or an ablating heat shield generates great charged particles, and when the density of the charged particles surpasses the plasma density at the frequency, the plasma sheath will black out the communication signal [6]. The plasma frequency $f_{p}$ is defined as

$$
f_{p}=\frac{1}{2 \pi} \sqrt{\frac{q^{2} n_{e}}{\varepsilon_{0} m_{e}}},
$$

where $q$ is the electron charge $\left(q=1.6 \times 10^{-19}\right.$ coulombs), $n_{e}$ is the electron number density per cubic meter (particles/ $\left.\mathrm{m}^{3}\right), m_{e}$ is the electron mass $\left(m_{e}=9.1 \times 10^{-31} \mathrm{~kg}\right)$, and $\varepsilon_{0}$ is the permittivity $\left(\varepsilon_{0}=8.85 \times 10^{-12} \mathrm{~F} / \mathrm{m}^{2}\right)$. So, when the UHF communication frequency, $f_{\mathrm{UHF}}=401 \mathrm{MHz}$, is greater than the plasma frequency $f_{p}$, the signal attenuation usually is negligible; otherwise, the signal attenuation should be considered. The signal attenuation is described by the amount attenuation per unit length, which is defined by [6]:

$$
\frac{\mathrm{dB}}{z_{p}}=\frac{54.6}{\lambda} \sqrt{\frac{f_{p}^{2}-f_{\mathrm{UHF}}^{2}}{f_{\mathrm{UHF}}^{2}}},
$$

where $\mathrm{dB}$ is the total attenuation, $z_{p}$ is the thickness of the plasma sheath, and $\lambda$ is the wavelength of the signal. Figure 2 shows the attenuation amount per unit length for UHF versus the electron number density. It can be seen that the signal strength decreases rapidly when the real electron number density reaches and exceeds the critical electron number density of UHF.

3.2. Communications Blackout Estimations. In this paper, the JPL Horton program numerically simulates the chemical equilibrium process. We assume that the Martian atmosphere model with a surface pressure of about $6.1 \mathrm{mb}$ and an argon-free simplification proportion, in which the proportion of $\mathrm{CO}_{2}$ is $96.5 \%$ and $\mathrm{N}_{2}$ is $3.5 \%$ in the JPL Horton program, and other assumptions are used as in the literatures $[4,6,21]$. To predict the communications blackout during Mars entry, the atmospheric relative velocity and altitude are shown in Figure 3, and the atmospheric density is shown in Figure 4, because the combination of the atmospheric relative velocity and atmospheric density during Mars entry 

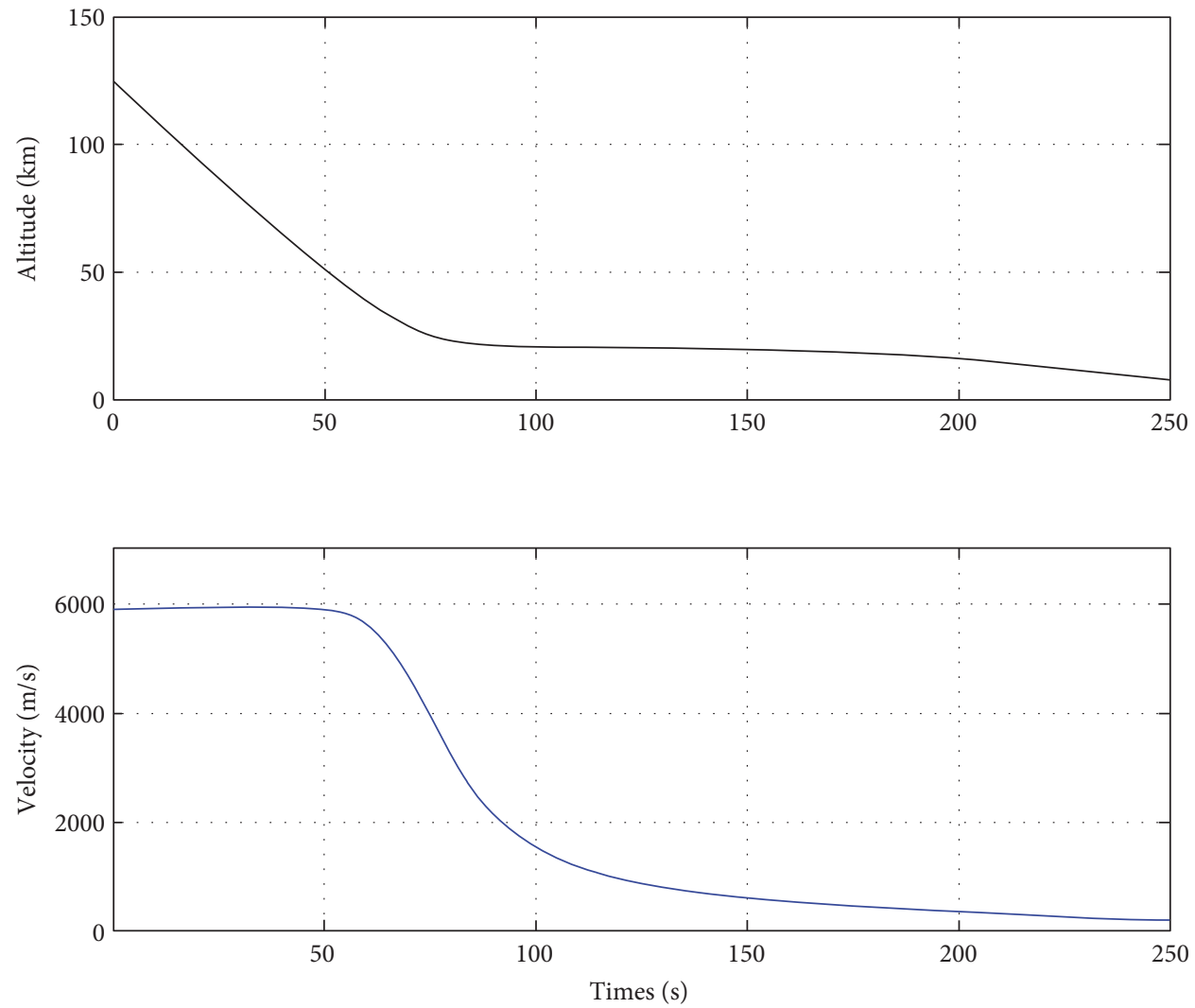

FIGURE 3: Atmospheric relative velocity and altitude of the entry vehicle.

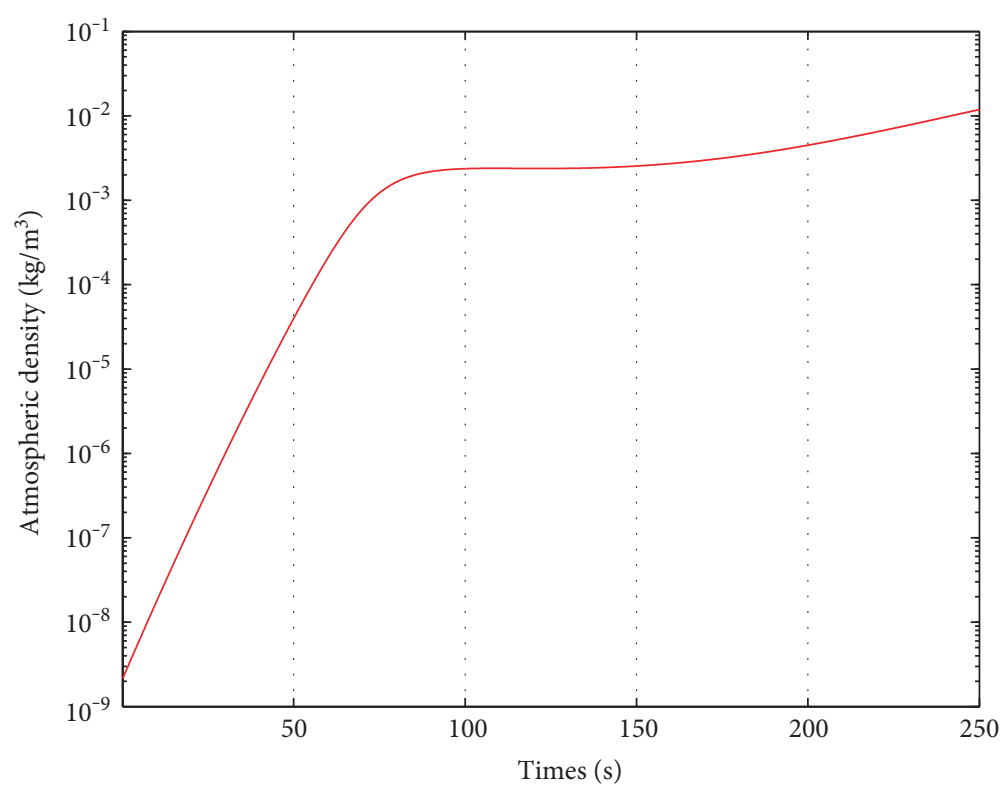

Figure 4: Atmospheric density during Mars entry.

is the main factor to affect the received signal degradation due to charged particles. Figure 3 shows the wake-region electron number density, the stagnation point electron number density, the blackout critical electron number density (blackout CEND), and the brownout critical electron number density (brownout CEND) for the $401 \mathrm{MHz}$ UHF. The blackout CEND for UHF is calculated by [6]

$$
n_{\text {blackout,crit }}=\frac{f_{\mathrm{UHF}}^{2}}{80.64 \times 10^{6}} \text {. }
$$




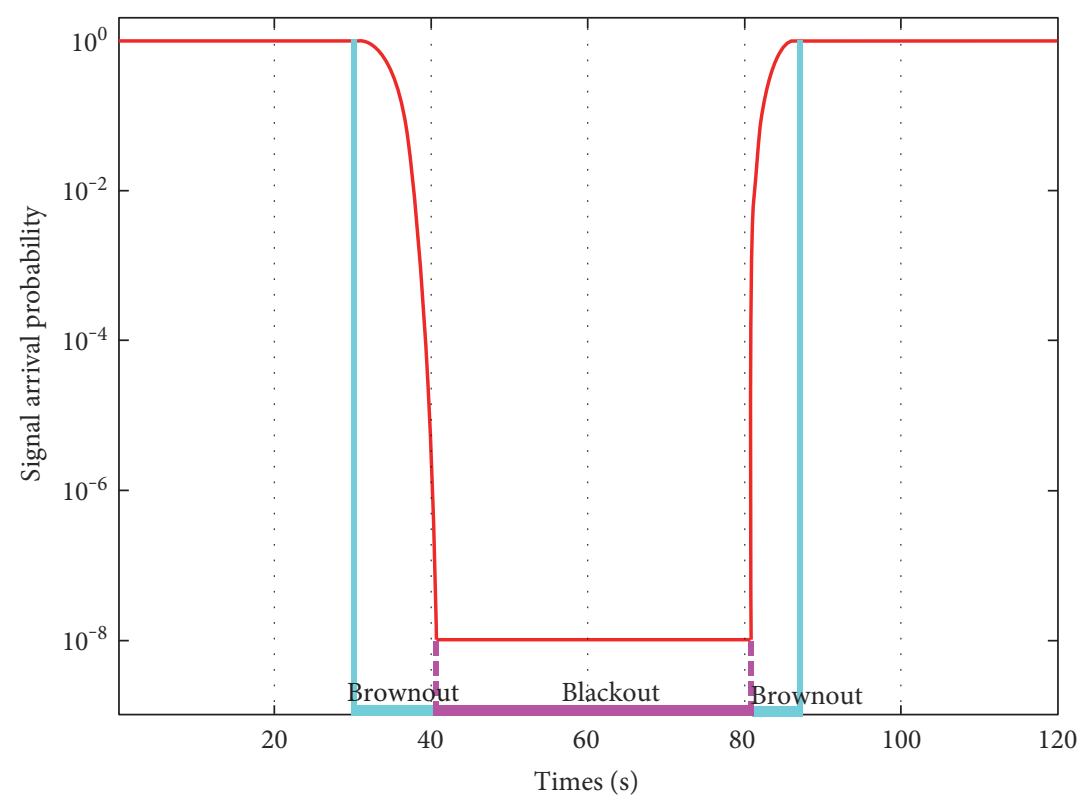

Figure 5: Probabilities of the received signal during Mars atmospheric entry.

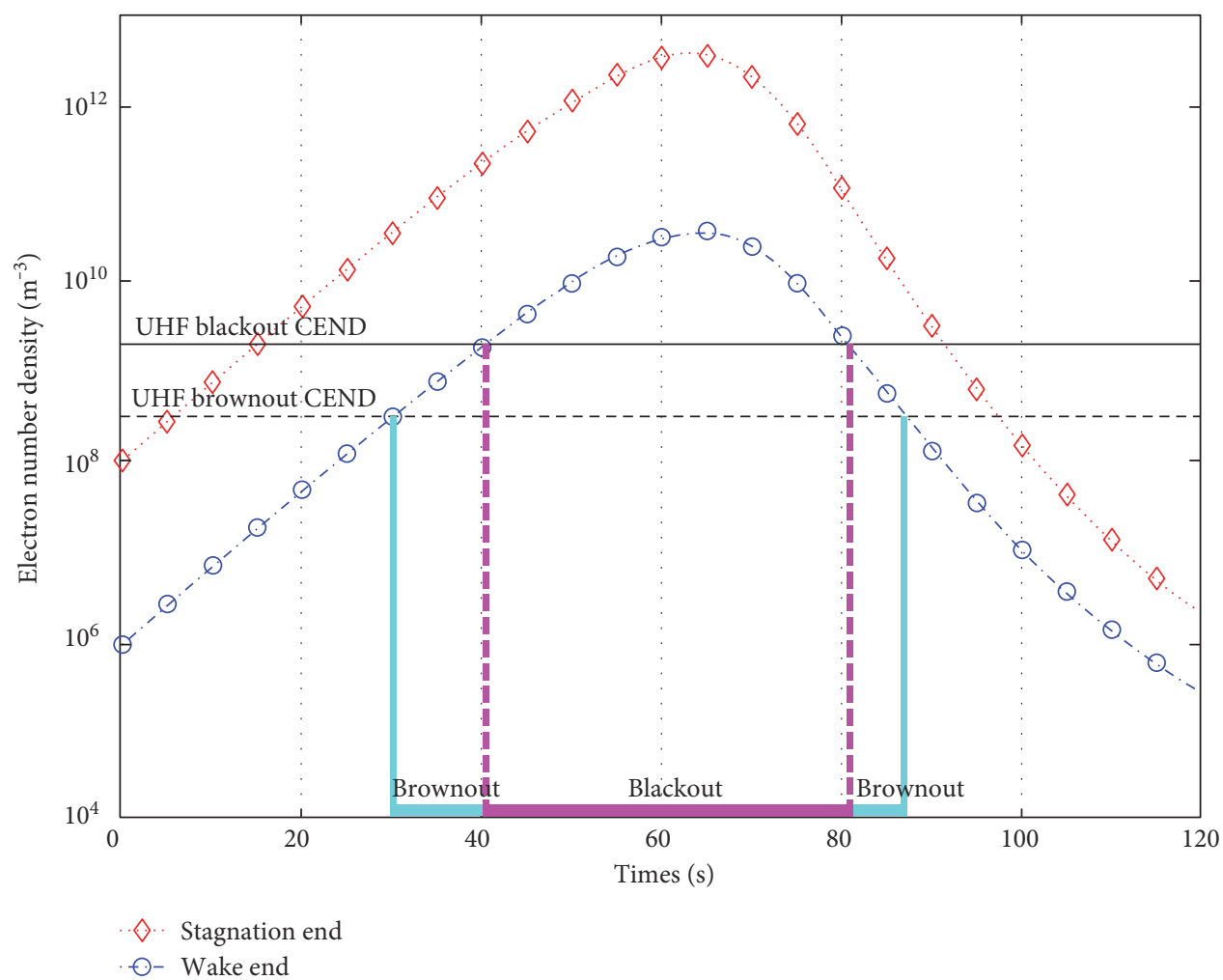

FIGURE 6: Predicted electron number density profiles (brownout: blue $30 \mathrm{~s} \sim 41 \mathrm{~s}, 81 \mathrm{~s} \sim 87 \mathrm{~s}$; red $6 \mathrm{~s} \sim 15 \mathrm{~s}, 91 \mathrm{~s} \sim 98 \mathrm{~s}$; blackout: blue $41 \mathrm{~s} \sim 81 \mathrm{~s}$; red $15 \mathrm{~s} \sim 91 \mathrm{~s})$.

The value of the blackout CEND for UHF is $n_{\text {blackout,crit }}=$ $1.9941 \times 10^{9}$ particles $/ \mathrm{m}^{3}$. The brownout CEND for UHF is estimated as $n_{\text {brownout,crit }}=3.08 \times 10^{8}$ particles $/ \mathrm{cm}^{3}$, which comes from Figure 5 in the literature [2], and is just less than an order of magnitude compared with the blackout CEND.
From Figure 6, it can be seen that the peak predicted stagnation point electron number density at $64 \mathrm{~s}$ past entry is about $3.69 \times 10^{12}$ particles $/ \mathrm{cm}^{3}$, and the peak predicted wake-region electron number density at this time is about $3.70 \times 10^{10}$ particles $/ \mathrm{cm}^{3}$. The likely worst case vehicle 
degradation period at UHF spans from $6 \mathrm{~s}$ to $98 \mathrm{~s}$ for the stagnation and from $30 \mathrm{~s}$ to $87 \mathrm{~s}$ for the wake region. The signal fades between $6 s \sim 15 s$ and $91 s \sim 98 s$ for the stagnation and between $30 \mathrm{~s} \sim 41 \mathrm{~s}$ and $81 \mathrm{~s} \sim 87 \mathrm{~s}$ for the wake region, and the signal is lost completely between $15 \mathrm{~s} \sim 91 \mathrm{~s}$ for the stagnation and between $41 \mathrm{~s} \sim 81 \mathrm{~s}$ for the wake region, similarly to the signal link data the period around plasma degradation in literature [2].

3.3. Signal Arrival Probabilities. When the real electron number density reaches and exceeds the critical electron number density of UHF, the signal strength will decrease rapidly, and then the communication quality between the entry vehicle and the preselected radio beacons will seriously fade or even black out. Morabito et al. pointed that the UHF relay links from MSL to the MRO and to the MEX suffered degradation, which is a combination of brownout and blackout [2], and the communication outage is approximately $70 \mathrm{~s}$ during Mars atmospheric entry. The received carrier-to-noise ratio (CNR) is recorded and processed by using the signal data from a bandwidth of $\pm 30 \mathrm{~Hz}$, which its undisturbed level is about $42 \mathrm{~dB}$, and its outage threshold level is $10 \mathrm{~dB}$, during Mars atmospheric entry. For the MSL-to-MRO signal link in the degradation period, the calculated CNR shows that the brownout period is between roughly $30 \mathrm{~s}$ and $40 \mathrm{~s}$, where the CNR decreases from about $42 \mathrm{~dB}$ to below $10 \mathrm{~dB}$ and $82 \mathrm{~s} 100 \mathrm{~s}$, where the CNR increases from below $10 \mathrm{~dB}$ to $42 \mathrm{~dB}$, and the blackout period is between $40 \mathrm{~s}$ and $80 \mathrm{~s}$, where the CNR drops below $10 \mathrm{~dB}$ as expected for a small period about $70 \mathrm{~s}$ [2].

For the brownout period, the electron number density is above this brownout CEND and below the blackout CEND for UHF, and at the same time, the CNR is also above its outage threshold level and below its undisturbed level, but possibly, communications blackout is deemed uncertain. So, the uncertain received navigation signals between the vehicle and the radio beacons with UHF will make the navigation data cannot arrive to its destination in time to be used for navigation data process. Here, the uncertain navigation measurement information is described as an intermittent measurement, in which the arrival of measurement is a Bernoulli process with parameter $0 \leq \beta \leq 1$ [2]. Next, we will define the probability of the arrival of navigation measurement information by using the electron number density.

The communication signal energy can be absorbed, reflected, refracted, and diffracted by the sheath of the charged particles around the hypersonic vehicle during Mars atmospheric entry. The magnitude of the signal will fade in otroposheric and ionospheric signal propagation, and it is approximately modeled as Rayleigh fading, which distributes to the Rayleigh distribution, and its probability density function and cumulative distribution function are given by [37].

$$
\begin{aligned}
& f(x)=\frac{x}{\tau^{2}} e^{-x^{2} / 2 \tau^{2}}, \quad x \geq 0, \\
& F(x)=1-e^{-x^{2} / 2 \tau^{2}}, \quad x \geq 0,
\end{aligned}
$$

where $\tau$ is the scale parameter of the distribution. Here, we assume that the signal strength in the brownout period during Mars atmospheric entry obeys the Rayleigh distribution, in which random variable is the electron number density. So, the probability of the arrival of navigation measurement information is calculated by the above Rayleigh distribution.

\section{Information Filter with Intermittent Measurement Design}

In this section, the intermittent measurement problem with multiple arrival probabilities is introduced into the traditional information filter (IF) [38, 39]. When the arrival probabilities of the measurements coming from navigation sensors are different, the information filter has a great advantage to deal with these intermittent measurement problems compared with the Kalman filter.

4.1. Problem Formulation. Consider the linear discretetime process and measurement models with intermittent measurements:

$$
\begin{aligned}
& \mathbf{x}_{k}=\boldsymbol{\Phi}_{k / k-1} \mathbf{x}_{k-1}+\mathbf{w}_{k-1}, \\
& \mathbf{z}_{k}=\Theta_{k} \mathbf{H}_{k} \mathbf{x}_{k}+\mathbf{v}_{k},
\end{aligned}
$$

where $\mathbf{x}_{k} \in \mathfrak{R}^{n}$ is a state vector and $\boldsymbol{\Phi}_{k / k-1}$ is the state transition matrix. $\mathbf{z}_{k}=\left[\left(\mathbf{z}_{k}^{1}\right)^{T},\left(\mathbf{z}_{k}^{2}\right)^{T}, \ldots,\left(\mathbf{z}_{k}^{N}\right)^{T}\right]^{T} \in \mathfrak{R}^{m}$ is a measurement vector, and $\mathbf{H}_{k}$ is the state measurement matrix. For every sensor, the measurement equation is

$$
\mathbf{z}_{i, k}=\lambda_{i, k} \mathbf{H}_{i, k} \mathbf{x}_{k}+\mathbf{v}_{i, k} .
$$

$\Theta_{k}$ is defined as $\Theta_{k}=\operatorname{diag}\left[\lambda_{1, k}, \lambda_{2, k}, \ldots, \lambda_{N, k}\right]$, in which random variable $\lambda_{i, k} \in \mathfrak{R}$ is a Bernoulli distributed sequence taking on values of 0 and 1 with

$$
\begin{aligned}
& \operatorname{Prob}\left\{\lambda_{i, k}=1\right\}=\beta_{i, k}, \\
& \operatorname{Prob}\left\{\lambda_{i, k}=0\right\}=1-\beta_{i, k},
\end{aligned}
$$

where $\beta_{i, k}$ is a known time-varying positive scalar satisfying $0 \leq \beta_{i, k} \leq 1$. If $t \neq s, \lambda_{t, k}$ is independent of $\lambda_{s, k}$, and $\lambda_{i, k}$ is assumed to be independent of $\mathbf{x}_{0}, \mathbf{w}_{k}$, and $\mathbf{v}_{i, k} \cdot \mathbf{w}_{k} \in \mathfrak{R}^{n}$ is independent zero-mean Gaussian noise processes with covariance $\mathbf{Q}_{k}$. The measurement noise $\mathbf{v}_{i, k} \in \Re^{m}$ is defined as

$$
p\left(\mathbf{v}_{i, k} \mid \lambda_{i, k}\right)= \begin{cases}\mathbf{N}\left(0, \mathbf{R}_{i, k}\right), & \lambda_{i, k}=1, \\ \mathbf{N}\left(0, \sigma_{i}^{2} \mathbf{I}\right), & \lambda_{i, k}=0,\end{cases}
$$

where $\sigma_{i}^{2} \mathbf{I}$ is the variance of the measurement at time $k$. If $\lambda_{i, k}$ is 0 for $\sigma_{i}^{2} \mathbf{I}$, and $\lambda_{i, k}$ is 1 for $\mathbf{R}_{i, k}$. When the real measurement does not arrive, the given variance of the measurement is taken as the limiting case of $\sigma_{i} \rightarrow \infty$ [22].

Here, the aforementioned measurement (13) represents intermittent measurements, missing measurements, or uncertain observations, in which the arrival of a measurement is the above Bernoulli distribution [23, 40, 41]. 
4.2. Traditional Information Filter. The information filter is the information form of the Kalman filter. The information matrix, $\mathbf{P}^{-1}$, is defined as the inverse of the covariance matrix $\mathbf{P}$ in the Kalman filter. In the information filter, some propagation equations must be rederived by using the ShermanMorrison-Woodbury matrix inversion lemma [39]

$$
(A+B C D)^{-1}=A^{-1}-A^{-1} B\left(D A^{-1} B+C^{-1}\right)^{-1} D A^{-1} .
$$

Here, the probability $\beta_{i, k}$ of the random variable $\lambda_{i, k}$ in (15) for all $i$ and $k$ is assumed as $\beta_{i, k}=1$, and this implies that the measurement arrives to its destination in time to be used for filtering. So, the measurement equation of the information filter is given by

$$
\mathbf{z}_{k}=\mathbf{H}_{k} \mathbf{x}_{k}+\mathbf{v}_{k} .
$$

Based on the process and measurement models in (12) and (19), the information is concluded as follows [22, 39]. The time update equations are

$$
\begin{aligned}
\widehat{\mathbf{x}}_{k / k-1} & =\boldsymbol{\Phi}_{k / k-1} \widehat{\mathbf{x}}_{k-1}, \\
\mathbf{P}_{k / k-1}^{-1} & =\mathbf{A}_{k-1}-\mathbf{A}_{k-1}\left(\mathbf{A}_{k-1}+\mathbf{Q}_{k-1}^{-1}\right)^{-1} \mathbf{A}_{k-1},
\end{aligned}
$$

and the measurement update equations are

$$
\begin{aligned}
\mathbf{P}_{k}^{-1} & =\mathbf{P}_{k / k-1}^{-1}+\sum_{i=1}^{N} \mathbf{H}_{i, k}^{T} \mathbf{R}_{i, k}^{-1} \mathbf{H}_{i, k}, \\
\mathbf{K}_{i, k} & =\mathbf{P}_{k} \mathbf{H}_{i, k}^{T} \mathbf{R}_{i, k}^{-1}, \\
\widehat{\mathbf{x}}_{k} & =\widehat{\mathbf{x}}_{k / k-1}+\sum_{i=1}^{N} \mathbf{K}_{i, k}\left(\mathbf{z}_{k}-\mathbf{H}_{i, k} \widehat{\mathbf{x}}_{k / k-1}\right),
\end{aligned}
$$

where $\mathbf{A}_{k-1}=\boldsymbol{\Phi}_{k / k-1}^{-1} \mathbf{P}_{k-1}^{-1} \boldsymbol{\Phi}_{k / k-1}^{-T}$.

4.3. Information Filter with Intermittent Measurement Algorithm. As the Kalman filter with intermittent measurements in [22], the variance of the measurement can be reconstructed as

$$
\mathbf{R}_{i, k}^{*}=\lambda_{i, k} \mathbf{R}_{i, k}+\left(1-\lambda_{i, k}\right) \sigma_{i}^{2} \mathbf{I} .
$$

Substituting $\mathbf{R}_{i, k}$ in (21) and (22) by $\mathbf{R}_{i, k}^{*}$ gives

$$
\begin{aligned}
& \mathbf{P}_{k}^{-1}=\mathbf{P}_{k / k-1}^{-1}+\sum_{i=1}^{N} \mathbf{H}_{i, k}^{T}\left\{\lambda_{i, k} \mathbf{R}_{i, k}+\left(1-\lambda_{i, k}\right) \sigma_{i}^{2} \mathbf{I}\right\}^{-1} \mathbf{H}_{i, k}, \\
& \mathbf{K}_{i, k}^{*}=\mathbf{P}_{k} \mathbf{H}_{i, k}^{T}\left\{\lambda_{i, k} \mathbf{R}_{i, k}+\left(1-\lambda_{i, k}\right) \sigma_{i}^{2} \mathbf{I}\right\}^{-1} .
\end{aligned}
$$

When $\lambda_{i, k}=0, \sigma_{i}$ is taken as the limit of $\sigma_{i} \rightarrow \infty$. Then (25) and (26) become

$$
\begin{aligned}
\mathbf{P}_{k}^{-1} & = \begin{cases}\mathbf{P}_{k / k-1}^{-1}+\sum_{i=1}^{N} \lambda_{i, k} \mathbf{H}_{i, k}^{T} \mathbf{R}_{i, k}^{-1} \mathbf{H}_{i, k}, & \lambda_{i, k}=1, \\
\mathbf{P}_{k / k-1}^{-1}, & \lambda_{i, k}=0,\end{cases} \\
\mathbf{K}_{i, k}^{*} & = \begin{cases}\mathbf{P}_{k} \mathbf{H}_{i, k}^{T} \mathbf{R}_{i, k}^{-1}, & \lambda_{i, k}=1, \\
\mathbf{0}, & \lambda_{i, k}=0 .\end{cases}
\end{aligned}
$$

TABLE 1: Information filter with intermittent measurements.

\begin{tabular}{lc}
\hline State propagation & $\widehat{\mathbf{x}}_{k / k-1}=\mathbf{\Phi}_{k / k-1} \widehat{\mathbf{x}}_{k-1}$ \\
Covariance propagation & $\mathbf{P}_{k / k-1}^{-1}=\mathbf{A}_{k-1}-\mathbf{A}_{k-1}\left(\mathbf{A}_{k-1}+\mathbf{Q}_{k-1}^{-1}\right)^{-1} \mathbf{A}_{k-1}$ \\
Covariance update & $\mathbf{P}_{k}^{-1}=\mathbf{P}_{k / k-1}^{-1}+\sum_{i=1}^{N} \lambda_{i, k} \mathbf{H}_{i, k}^{T} \mathbf{R}_{i, k}^{-1} \mathbf{H}_{i, k}$ \\
Gain & $\mathbf{K}_{i, k}=\mathbf{P}_{k} \mathbf{H}_{i, k}^{T} \mathbf{R}_{i, k}^{-1}$ \\
State update & $\widehat{\mathbf{x}}_{k}=\widehat{\mathbf{x}}_{k / k-1}+\sum_{i=1}^{N} \lambda_{i, k} \mathbf{K}_{i, k}\left(\mathbf{z}_{k}-\mathbf{H}_{i, k} \widehat{\mathbf{x}}_{k / k-1}\right)$ \\
\hline
\end{tabular}

Comparing with (21), (22), and (23) in the traditional information filter, some formulas of the information filter with intermittent measurements (IFIM) are reconstructed as follows:

$$
\begin{aligned}
\mathbf{P}_{k}^{-1} & =\mathbf{P}_{k / k-1}^{-1}+\sum_{i=1}^{N} \lambda_{i, k} \mathbf{H}_{i, k}^{T} \mathbf{R}_{i, k}^{-1} \mathbf{H}_{i, k}, \\
\widehat{\mathbf{x}}_{k} & =\widehat{\mathbf{x}}_{k / k-1}+\sum_{i=1}^{N} \lambda_{i, k} \mathbf{K}_{i, k}\left(\mathbf{z}_{k}-\mathbf{H}_{i, k} \widehat{\mathbf{x}}_{k / k-1}\right) .
\end{aligned}
$$

Note that the gain $\mathbf{K}_{i, k}$ in (28) and (29) has the same gain compared with the classical information filter. The IFIM algorithm is summarized in Table 1.

\section{Navigation Results}

Numerical simulations are carried out to analyze the performance of the proposed IFIM navigation algorithm during Mars atmospheric entry, when the arrivals of partial measurements between the orbiters and the vehicle by using UHF signal in the brownout period is described by a Bernoulli distributed sequence with a continuous probability. The state variables of the vehicle during Mars atmospheric entry are defined as $\mathbf{x}=\left[\begin{array}{llllll}r & \theta & \lambda & v & \gamma & \psi\end{array}\right]^{T}$, and the measurement information includes the acceleration $\tilde{\mathbf{a}}$ and three ranges $\tilde{R}_{1}, \tilde{R}_{2}$, and $\tilde{R}_{3}$.

In this work, the Mars entry initial conditions and parameters are listed in Table 2. Some state variable errors are considered, which include an altitude error of $1 \mathrm{~km}$, a velocity error of $20 \mathrm{~m} / \mathrm{s}$, a longitude and latitude angle error of $0.02 \mathrm{deg}$, and azimuth and FPA errors of $1 \mathrm{deg}$. Furthermore, the reference atmospheric density, the ballistic coefficient, and the L/D ratio are set as an arbitrary $10 \%$ model parameter error. The bank angle $\phi$ is assumed to be zero. The IMU accelerometer bias $\mathbf{b}_{a}$ used in the simulation is set as $\mathbf{b}_{a}=[0.003,0.003,0.003] \mathrm{m} / \mathrm{s}^{2}$. We assumed that three orbiting beacons are available for the UHF signal communication during Mars atmospheric entry. The initial positions and velocities of three orbiters in the Marscentered Mars-fixed coordinate system are given in Table 3 $[13,19]$. The numerical simulation is terminated after approximately $250 \mathrm{~s}$. 
TABLE 2: Initial conditions and parameters during Mars entry.

\begin{tabular}{lcc}
\hline Initial parameters & Values & Standard deviation \\
\hline Altitude $r$ & $3522.20 \mathrm{~km}$ & $1 \mathrm{~km}$ \\
Velocity $v$ & $5900 \mathrm{~m} / \mathrm{s}$ & $20 \mathrm{~m} / \mathrm{s}$ \\
FPA $\gamma$ & $-16 \mathrm{deg}$ & $0.5 \mathrm{deg}$ \\
Longitude $\theta$ & $0.00 \mathrm{deg}$ & $0.02 \mathrm{deg}$ \\
Latitude $\lambda$ & $0.00 \mathrm{deg}$ & $0.02 \mathrm{deg}$ \\
Azimuth $\psi$ & $5.156 \mathrm{deg}$ & $0.5 \mathrm{deg}$ \\
Reference density $\rho_{0}$ & $2.0 \times 10^{-4} \mathrm{~kg} / \mathrm{m}^{3}$ & $2 \times 10^{-5} \mathrm{~kg} / \mathrm{m}^{3}$ \\
L/D ratio & 0.156 & 0.016 \\
Ballistic coefficient $B$ & $0.016 \mathrm{~m}^{2} / \mathrm{kg}$ & $0.0016 \mathrm{~m}^{2} / \mathrm{kg}$ \\
\hline
\end{tabular}

TABle 3: Positions and velocities of orbiters.

\begin{tabular}{lcc}
\hline Beacon & Initial positions $(\mathrm{km})$ & Initial velocities $(\mathrm{m} / \mathrm{s})$ \\
\hline $\begin{array}{l}\text { Orbiting } \\
\text { beacon 1 }\end{array}$ & $(780.44,13276.13,1267.20)$ & $(-317.7,-50,1697.2)$ \\
$\begin{array}{l}\text { Orbiting } \\
\text { beacon 2 }\end{array}$ & $(3492.70,3659.20,215.11)$ & $(-605.1,97,3232.8)$ \\
$\begin{array}{l}\text { Orbiting } \\
\text { beacon 3 }\end{array}$ & $(3927.85,-230.90,374.91)$ & $(93.6,3120.8,-584.1)$ \\
\hline
\end{tabular}

In this simulation, the communications blackout problem between the entry vehicle and the orbiters is considered as two parts, which are the brownout period and blackout period. Since the antennas used for UHF communication are preinstalled on the back shell of the entry vehicle as in Figure 1, the communication quality is affected by the electron number density in the wake-region. In Section 3.3, the probabilities of the arrival of navigation measurements are introduced by using the Rayleigh distribution with the electron number density for the wake region in the brownout period. The scale parameter $\tau$ of the Rayleigh distribution is set as $\tau=2.5$ in the simulation to characterize the received signal probabilities of the entry vehicle, and the probabilities of the received signal in brownout period during Mars atmospheric entry are shown in Figure 5. In addition, the probabilities of the received signal in blackout period are set as $10^{-8}$, which is a very small probability to receive signals in the blackout period as in literature [2].

Considering the communications brownout and blackout problem during Mars atmospheric entry, the numerical simulations are run for three navigation schemes. One is the default navigation scenario based on IMU measurement alone, because the IMU is the only available conventional sensor and other sensors are impaired by the vehicle heat shield during Mars atmospheric entry. Another one is the information filter integrated navigation scenario based on the IMU and range measurements without considering the loss of the range measurements when the communications suffered signal degradation during Mars atmospheric entry.
For this navigation scenario, the loss of the range measurement with a big order of magnitudes makes the measurement model seriously unmatch to generate large navigation errors or even diverge. For this reason, the navigation errors may be diverge in the numerical simulation for this second navigation scenario, when first communication signal cannot be received by the UHF antenna mounted on the vehicle back shell. So, the navigation results of the second scenario are not shown in this paper. The last one is the IFIM integrated navigation scenario based on the IMU and range measurements with arrival probabilities. When the range measurements between the vehicle and the orbiters cannot arrive to navigation compute center in time, the range measurement noise is set as infinity, which implies that the range measurement is not adopted and only information provided by the IMU is used.

Figure 7 shows the state estimate errors and its $3 \sigma$ bounds of the information filter for the default IMU navigation scenario. Figure 8 shows the state estimate errors and the $3 \sigma$ bounds of the IFIM for the IMU/orbiter integrated navigation scenario. It is shown that the state errors of the altitude, velocity, FPA and latitude of the information filter are not captured by their $3 \sigma$ covariance bounds. Figure 8 shows that the $3 \sigma$ covariance bounds of all the states can almost capture the corresponding errors. When the communication signals between the vehicle and the orbiters begin to degrade, and the range measurements from the UHF communications cannot arrive in time, the state estimation error covariance continues to increase, because only used IMU information is used. The covariance of the IFIM navigation algorithm reconverges again and stays small for the reminder of the numerical simulation some time after reacquisition of the range measurements when the communications blackout disappears.

The state estimate root mean square error (RMSE) with logarithmic scales in 1000 time simulations is introduced to compare the performance of both the traditional information filter for default IMU navigation scenario and the IFIM for IMU/orbiter integrated navigation scenario. The RMSEs of both navigations are plotted in Figure 9. The all state RMSEs of the IFIM for IMU/orbiter integrated navigation scenario are obviously smaller than those of the traditional information filter for the default IMU navigation scenario; the state RMSEs of altitude and velocity using the traditional information filter are too large to meet the requirements of the future Mars pinpoint landing. For IMU/orbiter integrated navigation scenario with the intermittent range measurements in Mars communications brownout and blackout periods, the IFIM algorithm deals with the measurement partial loss problem very well, and the final navigation accuracy can meet future Mars missions.

\section{Conclusions}

An integrated navigation scenario with the probabilities of the radio range measurements in the communications brownout period during the Mars atmospheric entry by using the proposed IFIM is designed. The UHF signal attenuation per unit length versus electron number density 

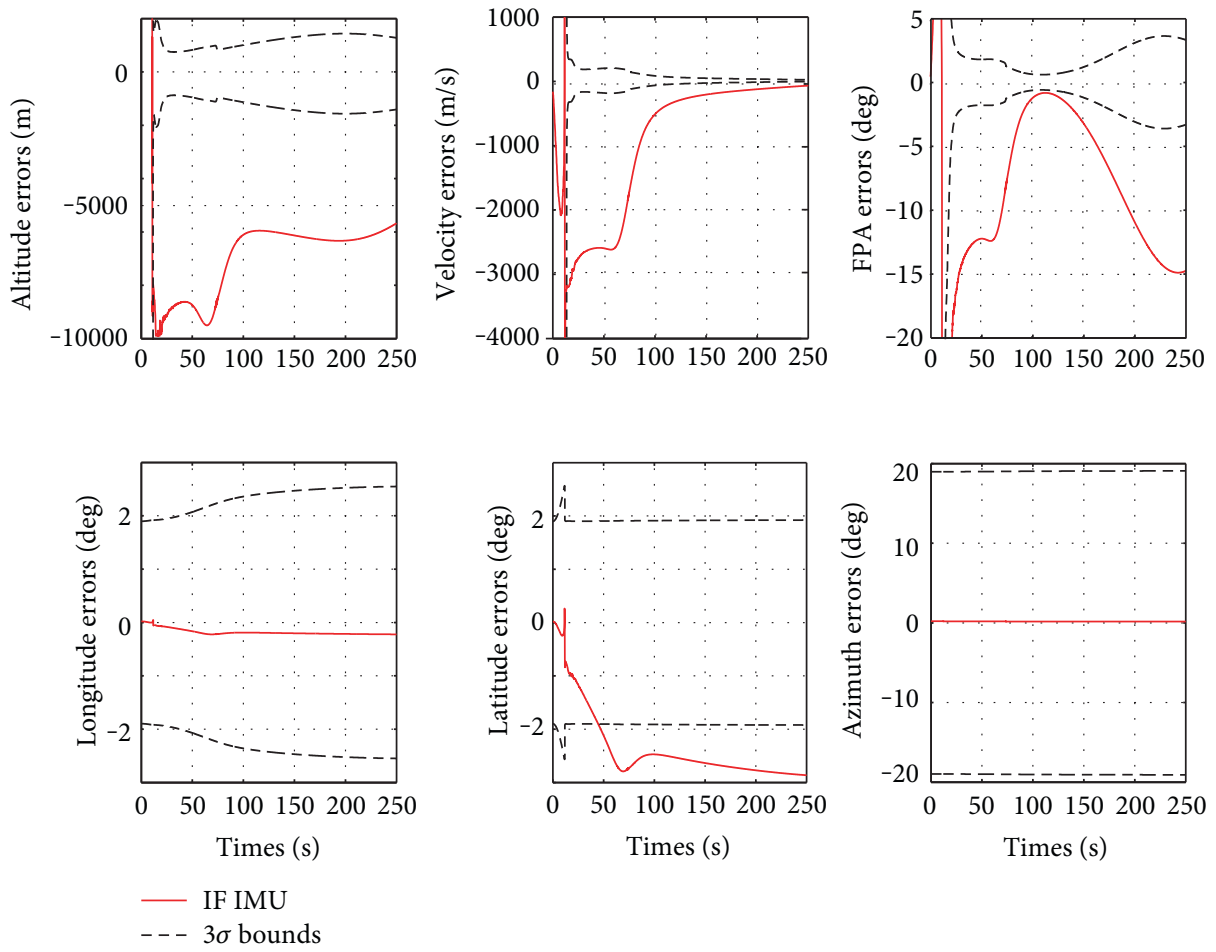

FIgURE 7: State estimate errors and $3 \sigma$ bounds for the default IMU navigation scenario.
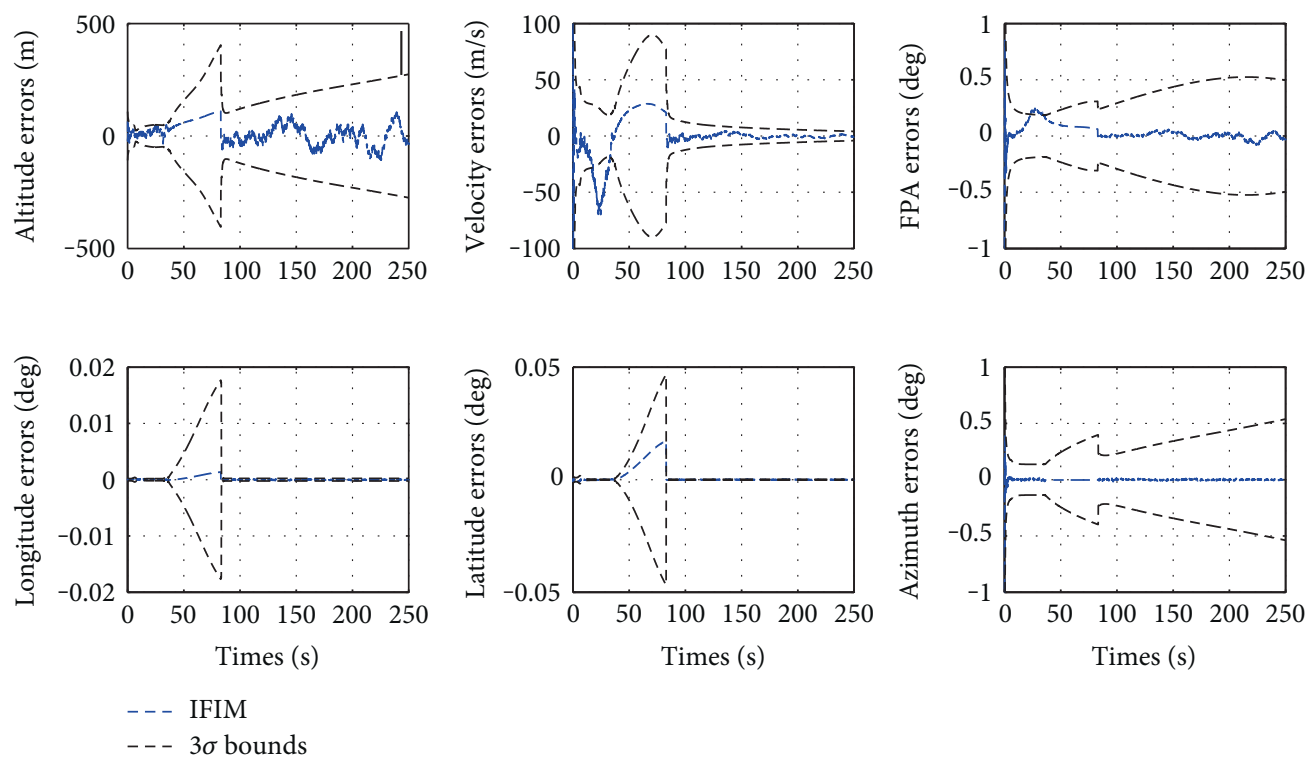

FIGURE 8: State estimate errors and $3 \sigma$ bounds of IFIM for IMU/orbiter integrated navigation scenario.

is analyzed. The vehicle will suffer about $17 \mathrm{~s}$ brownout period and about $40 \mathrm{~s}$ blackout period with an altitude and velocity profiles. In the brownout period, the intermittent range measurements are modeled by a Bernoulli distributed sequence with different arrival probabilities, which distributed to Rayleigh distribution of the electron number density around the entry vehicle for the UHF. To deal with the potential missing range measurements and the IMU information, the information filter with multiple intermittent measurements is derived. In fact, the measurements are partial intermittent in simulation, because the IMU measurements always arrive in time and only the range measurements are lost at a certain probability. The numerical simulations under the integrated navigation 

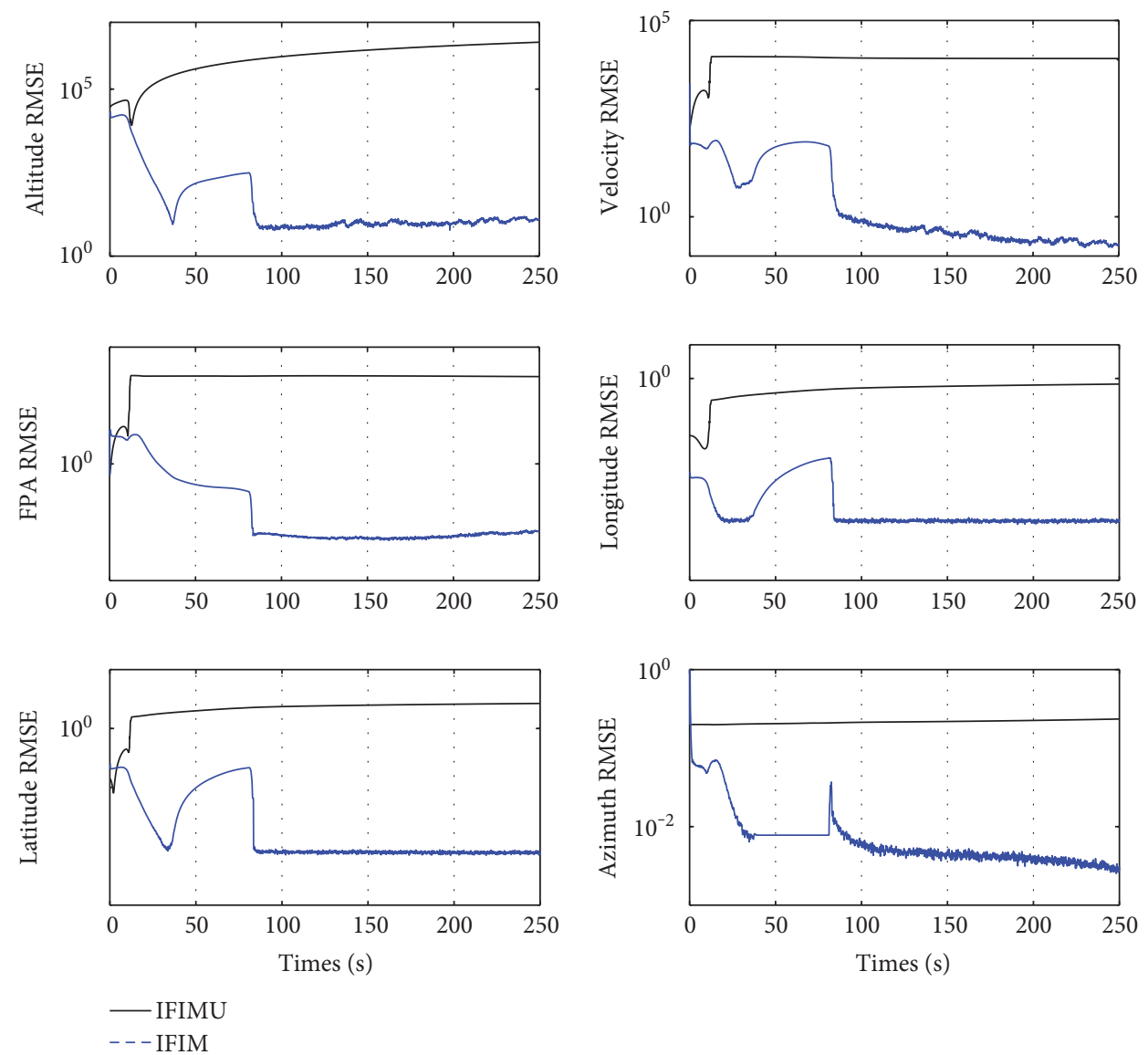

FIgURE 9: RMSE.

scheme based on IMU and three orbiters are carried out. The results show that the IFIM navigation filtering has a good performance to deal with partial intermittent range measurements in the communications brownout period.

\section{Conflicts of Interest}

The authors declare no conflict of interest.

\section{Acknowledgments}

The work described in this paper was supported by the National Nature Science Foundation of China (Grant nos. 61603346 and 61603347), the Fundamental Research Programs for the Provincial Universities (Grant no. 15KYYWF01), the key research projects of Henan higher education institutions (Grant no. 18A413003), and the Doctor Startup Funds Foundation of Zhengzhou University of Light Industry (Grant no. 2015BSJJ026). The authors fully appreciate the financial support.

\section{References}

[1] D. W. Way, R. W. Powell, A. Chen et al., "Mars science laboratory: entry, descent, and landing system performance," in 2007 IEEE Aerospace Conference, pp. 1-19, Big Sky, MT, USA, March 2007.
[2] D. D. Morabito, B. Schratz, K. Bruvold, P. Ilott, K. Edquist, and A. D. Cianciolo, "The Mars Science Laboratory EDL communications brownout and blackout at UHF," Interplanetary Progress Report, 2014.

[3] D. D. Morabito and K. T. Edquist, "Communications blackout predictions for atmospheric entry of Mars Science Laboratory," in 2005 IEEE Aerospace Conference, pp. 489-500, Big Sky, MT, USA, March 2005.

[4] T. E. Horton, The JPL Thermochemistry and Normal Shock Computer Program, Jet Propulsion Laboratory, California Institute of Technology, Pasadena, CA, USA, 1964.

[5] C. Park, J. Howe, R. Jaffe, and G. Chander, "Review of chemicalkinetic problems of future NASA missions, II: Mars entries," Journal of Thermophysics and Heat Transfer, vol. 8, no. 1, 1994.

[6] D. D. Morabito, "The spacecraft communications blackout problem encountered during passage or entry of planetary atmospheres," IPN Progress Report, 2002.

[7] B. C. Schratz, M. Soriano, P. Ilott, J. Shidner, A. Chen, and K. Bruvold, "Telecommunications performance during entry, descent, and landing of the Mars Science Laboratory," Journal of Spacecraft and Rockets, vol. 51, no. 4, pp. 1237-1250, 2014.

[8] S. Li and X. Q. Jiang, "Review and prospect of guidance and control for Mars atmospheric entry," Progress in Aerospace Sciences, vol. 69, pp. 40-57, 2014.

[9] S. Li and Y. M. Peng, "Radio beacons/IMU integrated navigation for Mars entry," Advances in Space Research, vol. 47, no. 7, pp. 1265-1279, 2011. 
[10] E. G. Lightsey, A. E. Mogensen, P. D. Burkhart, T. A. Ely, and C. Duncan, "Real-time navigation for Mars missions using the Mars network," Journal of Spacecraft and Rockets, vol. 45, no. 3, pp. 519-533, 2008.

[11] J. F. Levesque and J. d. Lafontaine, "Innovative navigation schemes for state and parameter estimation during Mars entry," Journal of Guidance, Control, and Dynamics, vol. 30, no. 1, pp. 169-184, 2007.

[12] J. L. Williams, P. R. Menon, and S. W. Demcak, "Mars Reconnaissance Orbiter Navigation Strategy for Mars Science Laboratory Entry, Descent and Landing Telecommunication Relay Support," in AIAA/AAS Astrodynamics Specialist Conference, AIAA 2012-4747, Minneapolis, MN, USA, August 2012.

[13] S. Li, X. Q. Jiang, and Y. F. Liu, "High-precision Mars entry integrated navigation under large uncertainties," Journal of Navigation, vol. 67, no. 2, pp. 327-342, 2014.

[14] S. Li, X. Q. Jiang, and Y. F. Liu, "Innovative Mars entry integrated navigation using modified multiple model adaptive estimation," Aerospace Science and Technology, vol. 39, pp. 403-413, 2014.

[15] Z. Yu, P. Cui, and S. Zhu, "Observability-based beacon configuration optimization for Mars entry navigation," Journal of Guidance, Control, and Dynamics, vol. 38, no. 4, pp. 643650, 2015.

[16] Z. Yu, P. Cui, and S. Zhu, "On the observability of Mars entry navigation using radiometric measurements," Advances in Space Research, vol. 54, no. 8, pp. 1513-1524, 2014.

[17] T. Lou, H. Fu, Z. Wang, and Y. Zhang, "Schmidt-Kalman filter for navigation biases mitigation during Mars entry," Journal of Aerospace Engineering, vol. 28, no. 4, article 04014101, 2015.

[18] T. Lou, H. Fu, Y. Zhang, and Z. Wang, "Consider unobservable uncertain parameters using radio beacon navigation during Mars entry," Advances in Space Research, vol. 55, no. 4, pp. 1038-1050, 2015.

[19] T. Lou, Research on self-calibration filtering method of the dynamic model and measurement model during Mars entry, [Ph.D thesis], Beihang University, Beijing, 2015.

[20] T. Lou and L. Zhao, "Robust Mars atmospheric entry integrated navigation based on parameter sensitivity," Acta Astronautica, vol. 192, pp. 60-70, 2016.

[21] X. Wang and Y. Xia, "Navigation strategy with the spacecraft communications blackout for Mars entry," Advances in Space Research, vol. 55, no. 4, pp. 1264-1277, 2015.

[22] B. Sinopoli, L. Schenato, M. Franceschetti, K. Poolla, M. I. Jordan, and S. S. Sastry, "Kalman filtering with intermittent observations," IEEE Transactions on Automatic Control, vol. 49, no. 9, pp. 1453-1464, 2004.

[23] Z. Wang, F. Yang, D. W. Ho, and X. Liu, "Robust finitehorizon filtering for stochastic systems with missing measurements," IEEE Signal Processing Letters, vol. 12, no. 6, pp. 437-440, 2005.

[24] M. Hadidi and S. C. Schwartz, "Linear recursive state estimators under uncertain observations," IEEE Transactions on Automatic Control, vol. 24, no. 6, pp. 944-948, 1979.

[25] F. Reali and G. B. Palmerini, "Evaluation of GNC strategies for terminal descent on solar system bodies," American Institute of Aeronautics and Astronautics, vol. 6855, pp. 20-23, 2007.

[26] T. De Carli Foletto and U. Franco Moreno, "On the performance of unscented Kalman filter with intermittent observations," in 2014 12th IEEE International Conference on Industrial Informatics (INDIN), pp. 660-665, Porto Alegre, Brazil, July 2014.

[27] F. Yang, Z. Wang, D. W. Ho, and M. Gani, "Robust Ho control with missing measurements and time delays," IEEE Transactions on Automatic Control, vol. 52, no. 9, pp. 16661672, 2007.

[28] J. Lygeros, D. N. Godbole, and S. Sastry, "Verified hybrid controllers for automated vehicles," IEEE Transactions on Automatic Control, vol. 43, no. 4, pp. 522-539, 1998.

[29] J. Nilsson, Real-Time Control Systems with Delays, [Ph.D thesis], Lund institute of Technology, Lund, Sweden, 1998.

[30] J. Hu, Z. Wang, H. Gao, and L. K. Stergioulas, "Extended Kalman filtering with stochastic nonlinearities and multiple missing measurements," Automatica, vol. 48, no. 9, pp. 20072015, 2012.

[31] X. Liu and A. Goldsmith, "Kalman filtering with partial observation losses," in 2004 43rd IEEE Conference on Decision and Control (CDC) (IEEE Cat. No.04CH37601), pp. 4180-4186, Nassau, Bahamas, December 2004.

[32] M. K. Lockwood, R. W. Powell, and C. Graves, "Entry system design considerations for Mars landers," in AAS Guidance and Control Conference, AAS 01-023, Breckenridge, CO, USA, 2001.

[33] S. Rousseau, E. Perot, C. Graves, J. Masciarelli, and E. Queen, "Aerocapture guidance algorithm comparison campaign," in AIAA/AAS Astrodynamics Specialist Conference and Exhibit, AIAA 4822, Monterey, CA, USA, August 2002.

[34] R. A. Boehmer, Navigation Analysis and Design for Mars Entry, [Ph.D thesis], Massachusetts Institute of Technology, Cambrigde, MA, USA, 1999.

[35] D. F. Spencer, "An evaluation of the communication blackout problem for a blunt Mars-entry capsule and a potential method for the elimination of blackout," JPL Technical Report 32-594, California Institute of Technology, 1964.

[36] S. Sgubini and G. B. Palmerini, "Design of descent trajectories in atmosphere with respect to engineering constraints," in 2004 IEEE Aerospace Conference Proceedings (IEEE Cat. No.04TH8720), Big Sky, MT, USA, March 2004.

[37] T. S. Rappaport, Wireless Communications: Principles and Practice, vol. 2, Prentice Hall PTR, Upper Saddle River, NJ, USA, 1996.

[38] P. Quan, Z. Yongmei, L. Yan, Y. Feng, and W. Xiaoxu, Multi-Source Information Fusion Theory and Its Applications, Tsinghua University Press, Beiijing, 2013.

[39] J. L. Crassidis and J. L. Junkins, Optimal Estimation of Dynamic Systems, Chapman \& Hall \CRC Press, Boca Raton, FL, USA, 2012.

[40] G. Chen, "A simple treatment for suboptimal Kalman filtering in case of measurement data missing," IEEE Transactions on Aerospace and Electronic Systems, vol. 26, no. 2, pp. 413415, 1990.

[41] W. NaNacara and E. E. Yaz, "Recursive estimator for linear and nonlinear systems with uncertain observations," Signal Processing, vol. 62, no. 2, pp. 215-228, 1997. 


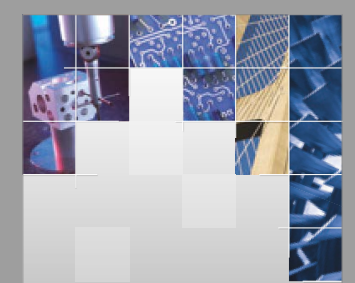

\section{Enfincering}
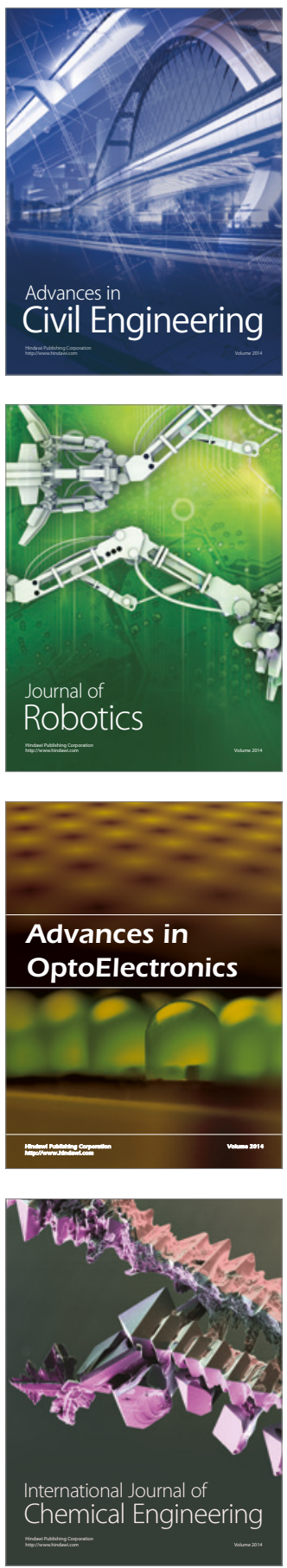

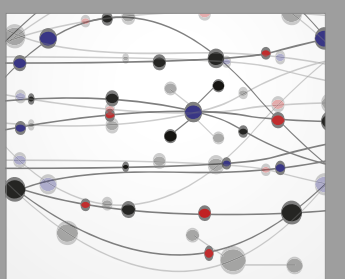

The Scientific World Journal

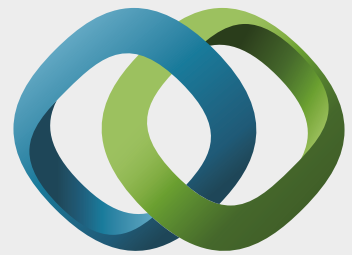

\section{Hindawi}

Submit your manuscripts at

https://www.hindawi.com
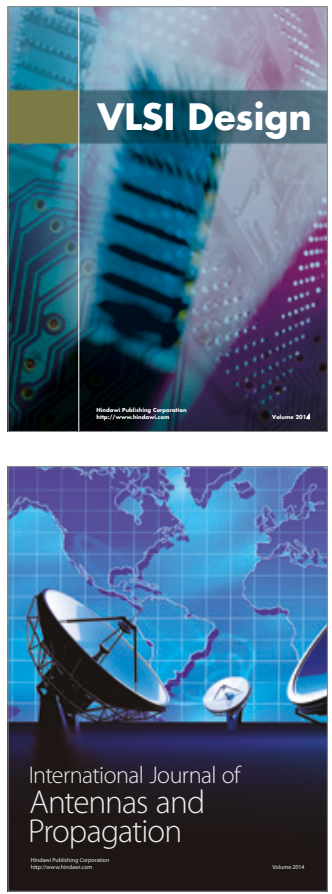

\section{Rotating}

Machinery
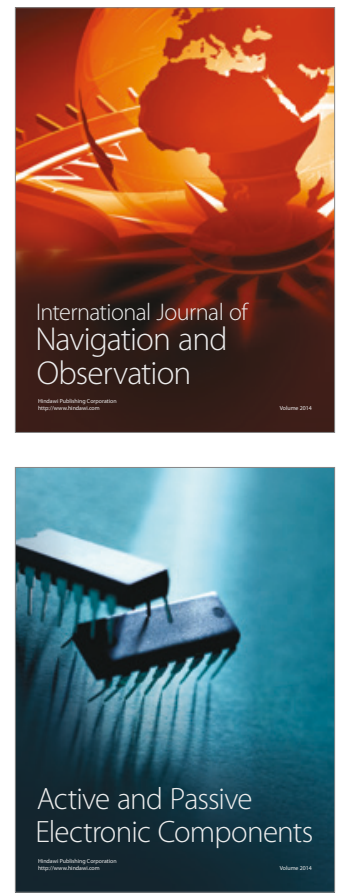
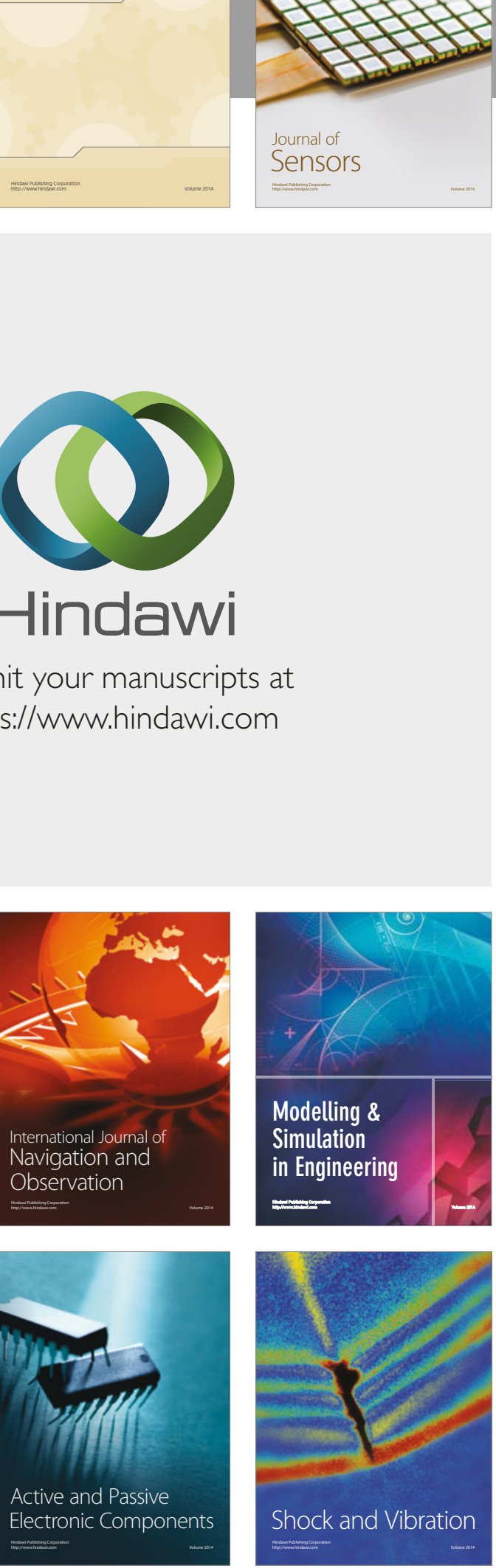
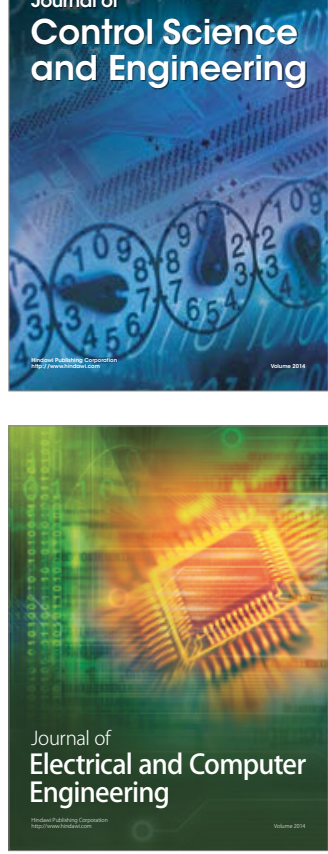

Distributed

Journal of

Control Science

and Engineering
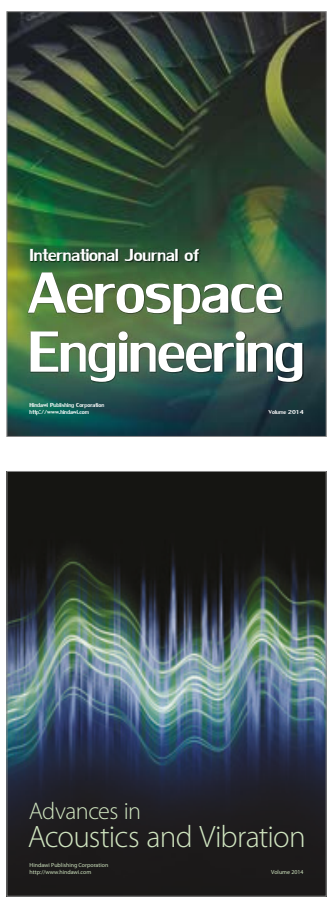

Sensor Networks 